\title{
ЭКСПЕРИМЕНТАЛЬНОЕ ИССЛЕДОВАНИЕ ПРОЦЕССА ГИБКИ ЛИСТОВ НА РОЛИКОВОЙ МАШИНЕ
}

В современной металлообрабатывающей промышленности существенное место занимает производство труб, обечаек и цистерн, подобного рода изделия используются повсеместно в нефтегазовой отрасли, энергетике, машиностроении и других отраслях. Существует несколько способов производства: прошивка кованой заготовки, прокатка, редуцирование, волочение, вальцовка и др. Все эти методы нашли свое применение в массовом производстве, которое характеризуется применением дорогих станов и технологической оснастки. В мелкосерийном же производстве использование этих методов нецелесообразно, поэтому для производства небольших партий труб и изделий нестандартного типоразмера (цистерны, обечайки) применяют вальцовку путем гибки листового проката в валках или роликах, как более универсальный и недорогой метод производства (рис. 1). В валковых листогибочных машинах гибка листа проводится между тремя или четырьмя вращающимися валками, расположенными в шахматном порядке [1-3].
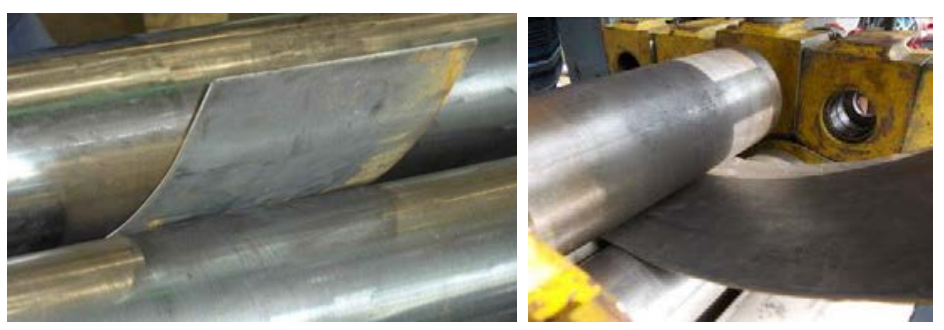

Рис. 1. Процесс вальцевания металла

С помощью соответствующего размещения валков по отношению друг к другу лист может быть изогнут на любой радиус, но не менее радиуса среднего валка. Наибольшая кривизна изгиба, получаемая листом за один пропуск, ограничивается силой сцепления приводных валков с листом. По этой причине сгибание на малые радиусы выполняют за несколько последовательных пропусков, причем после каждого пропуска увеличивают прогиб листа. Актуальность работы и ее практическое направление определяются необходимостью повышения технико-экономических показателей процесса гибки из листов цилиндрических изделий, осуществляемого на основе повышения степени научной обоснованности принятых технических решений.

Значительный вклад в развитие теории и практики производства профилированного листа внесли В. И. Давыдов, М. П. Максаков, Г. Я. Гун, П. И. Полухин, Г. А. Смирнов-Аляев, К. Н. Богоявленский, А. К. Григорьев, И. С. Тришевський, В. В. Драгобецкий, а также целый ряд ученых и специалистов. Так, например, в своей теории В. И. Давыдов и М. П. Максаков принимают, что поперечные сечения заготовки находятся постоянно в плоскостях перпендикулярных направлению движения, а кромки остаются прямолинейными. Г.Я. Гун, П. И. Полухин и Г. А. Смирнов-Аляев в своей математической модели процесса профилирования основываются на модели участка плавного перехода, для которого поперечные сечения заготовки остаются плоскими и перпендикулярными оси профилирования.

Наиболее точным из методов исследования процессов является метод конечных элементов. Наряду с этим, целый ряд вопросов, связанных с повышением эффективности промышленных условий реализации исследуемого процесса является неизученным или изученным недостаточно 
полно. Весьма немаловажным, помимо расчета энергосиловых параметров и проектирования собственно технологии, является дальнейшее совершенствование механического оборудования для профилирования ободьев колес.

Цель работы заключается в развитии численного математической модели для установления влияния факторов на протекание процесса, создании конечно-элементной модели для определения уровня энергосиловых параметров процесса, а также экспериментальном подтверждении адекватности математических моделей гибки листов и возможности их использования для практических расчетов при проектировании нового и совершенствовании существующего оборудования.

В соответствии с современными тенденциями развития научных подходов, теоретические исследования процесса гибки были выполнены с использованием численного математического моделирования напряженно-деформированного состояния (НДС) металла.

Математическое моделирование текущих по длине очага деформации процесса гибки листов геометрических характеристик было осуществлено на основе численной интерпретации известных инженерных методик $[4,5]$.

В основу математических моделей НДС гибки были возложены многочисленные рекуррентные решения конечно-разностной формы условий статического равновесия выделенных элементарных объемов очага деформации [6].

Также для оценки напряженно-деформированного состояния металла был использован метод конечных элементов, который в настоящее время является наиболее распространенным методом решения прикладных задач теории ОМД $[7,8]$. Необходимо отметить, что объем проведенных в лабораторных условиях исследований процесса профилирования обечаек не соответствует всему диапазону технологических режимов, используемых на практике. Недостаток материальных ресурсов, а также сжатые сроки привели к необходимости постановки и реализации численного эксперимента. С этой целью и была разработана математическая модель, основанная на использовании метода конечных элементов и позволяет корректно учесть весь диапазон граничных условий очага деформации, условий внешнего трения. Такая математическая модель имеет высокую информативность, поскольку ее результаты содержат полную информацию о геометрических характеристиках изделий, формируются, и распределение основных компонент напряженно-деформированного состояния металла по длине и толщине листа.

Математическая модель была реализована в пакете конечно-элементного моделирования Abaqus, который является универсальной программой общего назначения и широко применяется при расчете процессов ОМД $[9,10]$

Экспериментальные исследования результирующих геометрических характеристик листов при гибке были проведены на лабораторной экспериментальной 3-х роликовой листогибочной машине 100 х 105 х 250 ДГМА, общий вид которой представлен на рис. 2. Шаг машины 105 мм, диаметр бочки валков 100 мм, длина бочки валка 250 мм.

Гибка заготовки на экспериментальной машине 100 х 105 х 250 (рис. 2) происходит за счет упругопластического изгиба, создаваемого между тремя роликами, посредством смещения среднего ролика вниз. Рабочая клеть машины включает в себя узел станин, стянутые между собой стяжками. Верхние и нижние валковые узлы закреплены на выступах станин и опираются на нажимные винты, совмещенные с системой уравновешивания пружинного типа. Рабочим инструментом машины являются вальцы с диаметром бочки 100 мм, и длиной бочки 250 мм, установленные в подушках посредством двух шарикоподшипников радиального типа в каждой. В ходе проведения эксперимента формовке подвергались заготовки сечением 2,5 х 200 мм и длиной 1000 мм. В ходе проведения эксперимента было исследовано влияние перекрытия $W$ среднего ролика на остаточную кривизну $\chi_{\text {ocm }}$. Перекрытие среднего ролика $W$ составило 2.5 мм, 4 мм, 5 мм и 6 мм. Полученные результаты представлены на рис. 3. 

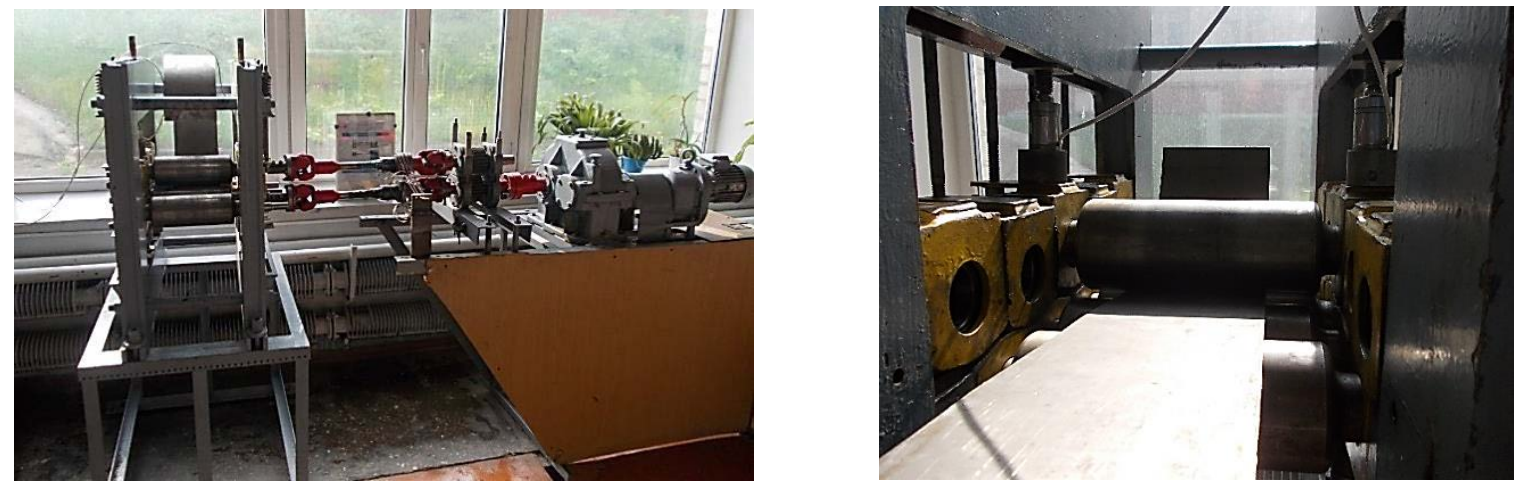

Рис. 2. Общий вид лабораторной экспериментальной машины 100 х 105 х 250
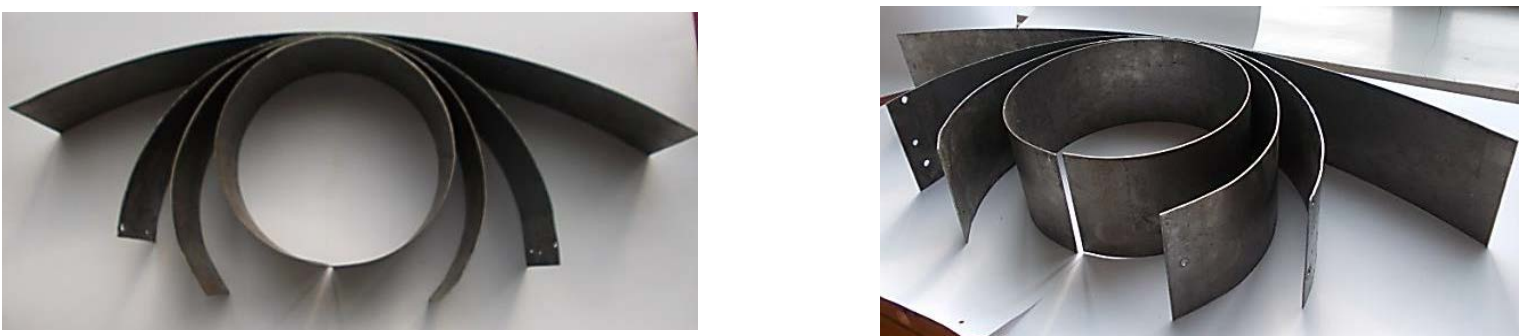

Рис. 3. Общий вид заготовок после формовки на лабораторной экспериментальной машине 100 × 105 × 250 ДГМА

В рамках анализа напряженно-деформированного состояния металла при его гибке на трехвалковых листогибочных машинах был выполнен расчет с использованием метода конечных элементов в системе Abaqus CAE. Выполнялась дискретизация объема (построение конечно-элементной сетки), занимаемого моделью, на элементарные области (конечные элементы). Для моделирования роликов были использованы трехмерные недеформируемые элементы. Моделирование листа происходило с использованием элементов S4R - четырех узловые, прямоугольные элементы оболочки с контролем разрушения [3].

В качестве примеров результатов численной реализации модели на рис. 4 представлены расчетные распределения основных компонент напряженно-деформированного состояния металла по высоте поперечного сечения листа, подвергаемого изгибу. Данные расчеты были выполнены применительно к изгибу листа толщиной $h=2.5$ мм из стали 08кп $\left(\sigma \mathrm{T} 0=240 \mathrm{H} / \mathrm{MM}^{2} ; \mathrm{a} 1=1133.4 \mathrm{H} / \mathrm{MM}^{2} ; \mathrm{a} 2=-1195.2 \mathrm{H} / \mathrm{MM}^{2} ; \mathrm{a} 3=525.6 \mathrm{H} / \mathrm{MM}^{2} ; \mathrm{E}=21 \cdot 10^{4} \mathrm{H} / \mathrm{Mm}^{2}\right)$. Величина перекрытия $W$ составила 2.5, 4.0, 5.0 и 6.0 мм. Распределения эквивалентных напряжений и деформаций при расчете методом конечных элементов представлены в графической форме в виде объемной деформированной модели на рис. 4.

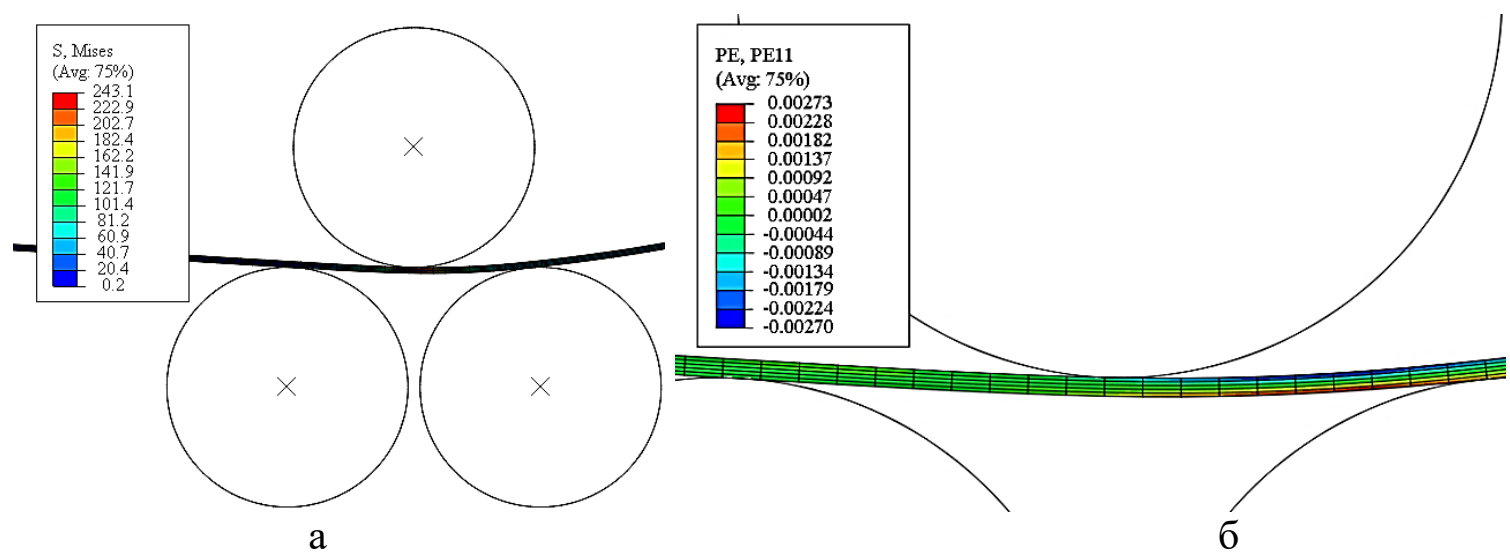

Рис. 4. Расчетные распределения эквивалентных напряжений (а) и деформаций (б) в листе при расчете процесса гибки в пакете конечно-элементного анализа Abaqus CAE 
Количественная и качественная оценка степени соответствия результатов экспериментальных исследований и конечно-элементной модели представлена на рис. 5. Обработка результатов как экспериментальных так и теоретических исследований была основана на выборке точек полученной формы образцов с различным шагом, геометрическом решении по определению радиуса изгиба заготовки и статистической обработке полученных результатов. При исследовании результатов конечно-элементной модели использовались координаты узлов сетки, расположенных по средней линии листа. Для визуализации результатов на рис. 5 представлены размеры радиусов листов полученных путем импортирования замеряемых точек в графическую среду AutoCAD посредством использования скрипт-файлов.

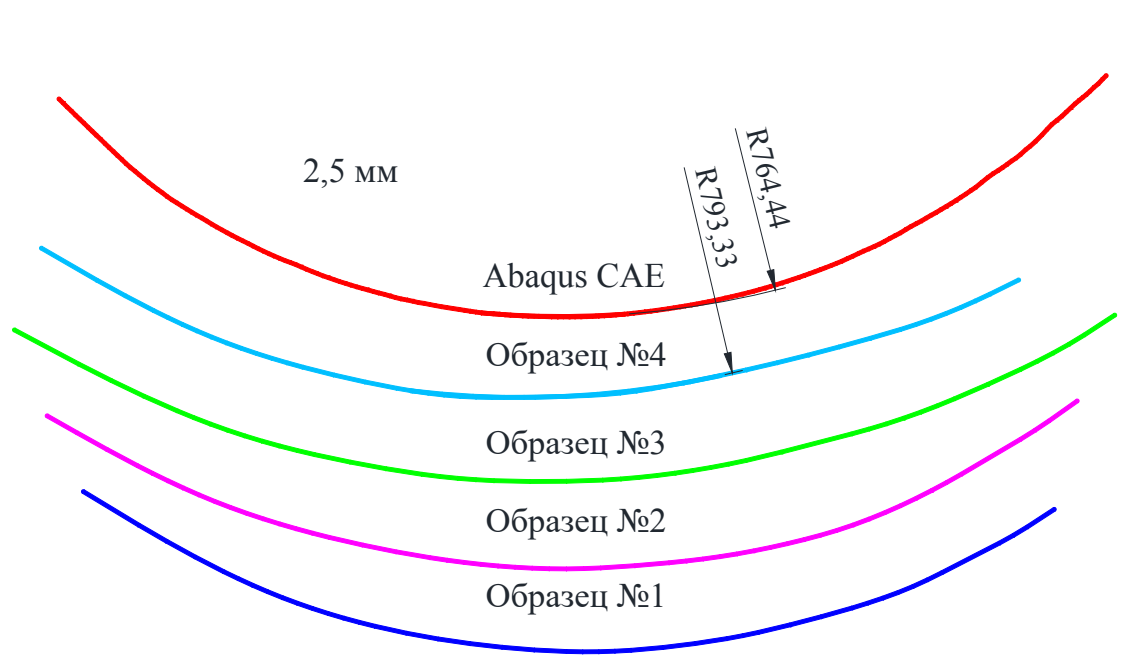

a

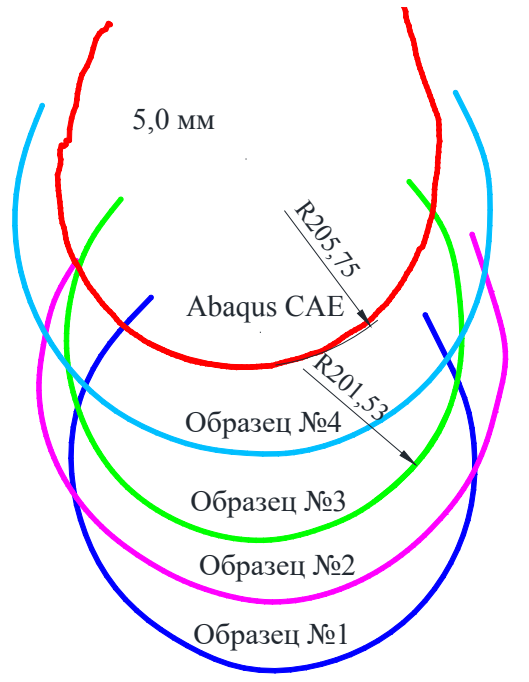

6
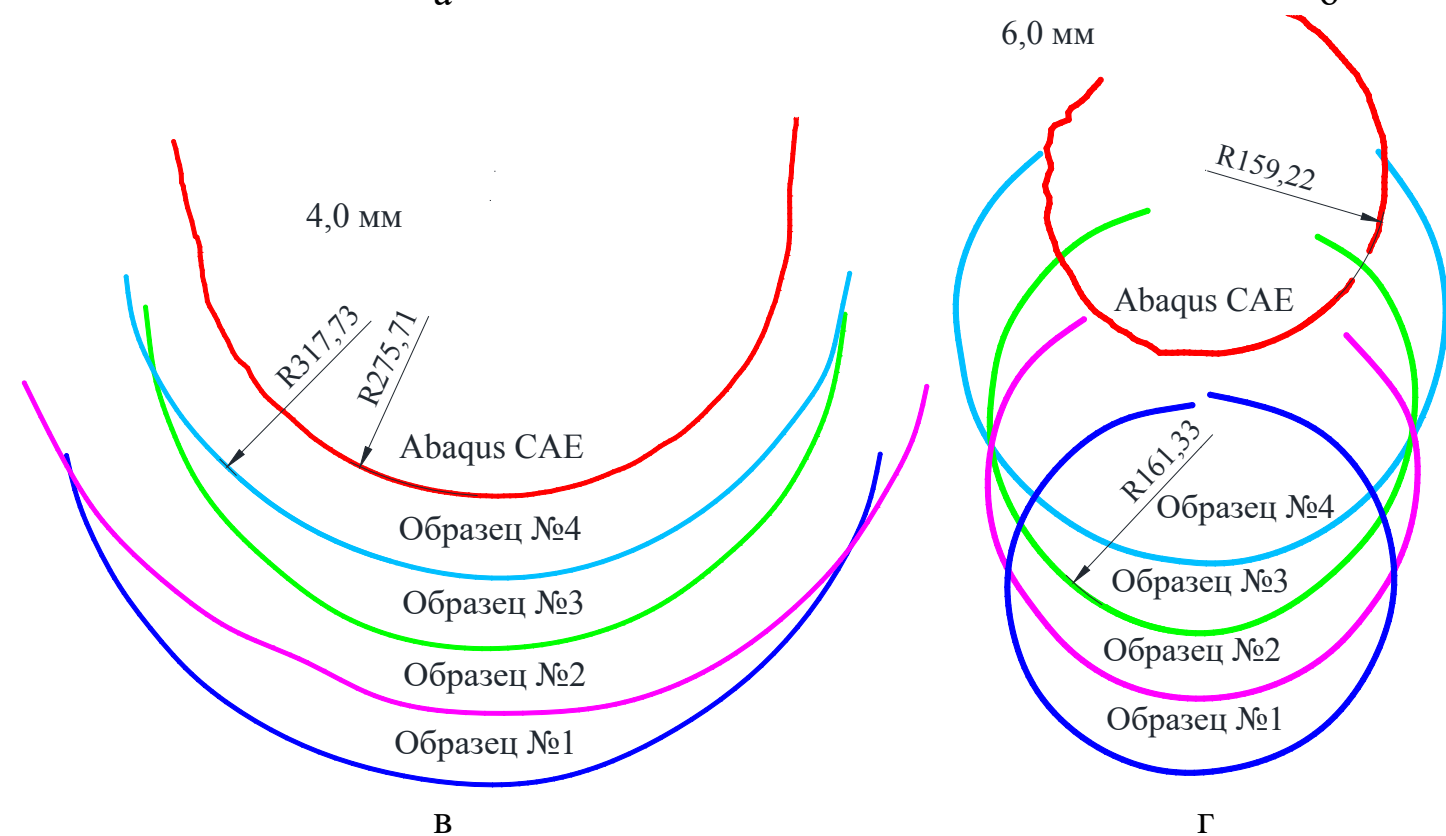

Рис. 5. Эмпирические и теоретические виды заготовки после гибки в зависимости от смещения среднего ролика: а - 2,5 мм; б-5,0 мм; в -4,0 мм; г-6,0 мм

Помимо конечно-элементной в работе была использована численная аналитическая модель на основе рекуррентного решения конечно-разностной формы условий статического равновесия выделенных элементарных объемов очага деформации [6]. Данная модель предпочтительнее при решении задач оптимизационного плана, например, при проектировании нового оборудования или при расчете технологических настроек. Сравнение результатов экспериментальных исследований и результатов используемых моделей представлены на рис. 6. 
На основе статистической обработки полученных массивов было установлено, что критерий Фишера при сравнении результатов экспериментов и численной аналитической модели составил - 0,949, а при сравнении экспериментов и конечно-элементной модели 0,931, что вполне удовлетворительно и подтверждает возможность использования аналитической модели для решения задач оптимизационного плана, а конечно-элементной - для исследования напряженно-деформированного состояния при совершенствовании процессов гибки листового металлопроката на роликовой гибочной машине.

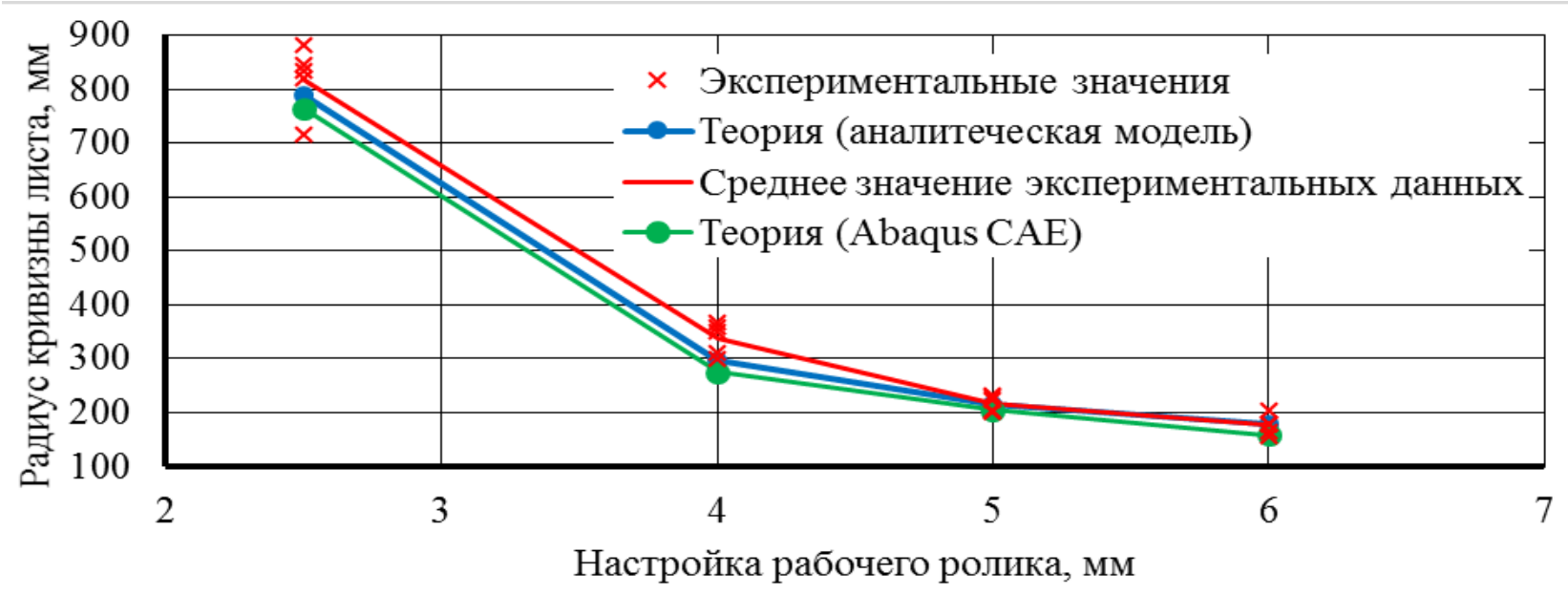

Рис. 6. Результаты экспериментальных и теоретических исследований процесса гибки листов

\section{ВЫВОДЫ}

В результате экспериментальных процесса гибки на лабораторной машине $100 \times 105$ х 250 получены эмпирические зависимости радиуса остаточной кривизны листа от технологических настроек машины. Эксперименты были проведены для листов из стали марки 08кп, толщиной 2,5 мм и шириной 205 мм. Величина перекрытия составляла от 2,5 до 6,0 мм. При перекрытии 6,0 мм заготовка принимала форму близкой к окружности. Также в работе было выполнено моделирование процесса гибки на основе конечно-элементной и аналитической модели. Оба типа моделей используются на практике для определения качественной картины процесса и получения количественных параметров в режиме реального времени. Обработка результатов как экспериментальных так и теоретических исследований была основана на выборке точек полученной формы образцов с различным шагом, геометрическом решении по определению радиуса изгиба заготовки и статистической обработке полученных результатов. При исследовании результатов конечно-элементной модели использовались координаты узлов сетки, расположенных по средней линии листа. В случае аналитической модели была использована относительная кривизна листа, получаемая непосредственно при расчетах. Сравнение полученных результатов с результатами разработанных конечно-элементной и численной аналитической моделей показали удовлетворительную сходимость результатов, при этом критерий Фишера при сравнении результатов экспериментов и численной аналитической модели составил - 0,949, а при сравнении экспериментов и конечно-элементной модели - 0,931, что подтверждает возможность использования как аналитической, так и конечно-элементной модели при совершенствовании процессов гибки листового металлопроката на роликовой гибочной машине.

\section{СПИСОК ИСПОЛЬЗОВАННОЙ ЛИТЕРАТУРЫ}

1. Мошнин Е. Н. Гибка и правка на ротационных машинах. Москва: Машиностроение, 1967. 272 с.

2. Мошнин Е. Н. Гибочные и правильные машиниы. Москва: МАШГИЗ. 1956. 252 с.

3. Пасечник Н. В. Машиностроительная энциклопедия. В 40 т. Т. 4, 5 Машины и агрегаты металлургического производства / под ред. В. М. Синицкого, Н. В. Пасечника. Москва: Машиностроение, 2000. 912 с. 
4. Руководство по гибке высокопрочной толстолистовой стали на валковых листогибочных машинах заводов металлоконструкций: руководство. ЦНИИПРОЕКТСТАЛЬКОНСТРУКЦИЯ. Москва, 1978. 61 с.

5. Самойлов В. А., Егоров А. Б. О взаимосвязи силовых факторов и кривизны в процессе деформирования проката растяжением с изгибом. Изв. вузов. Черная металлургия. 1989. № 7. С. 148-149.

6. Сатонин А. В. Численное конечно-разностное математическое моделирование напряженнодеформированного состояния металла при реализации различных технологических схем обработки давлением. Удосконалення процесів та обладнання обробки тиском в металургії і машинобудуванні: зб. наук. пр. Краматорськ: ДДМА, 2001. С. 559-564.

7. Зенкевич О., Морган К. Конечные элементы и аппроксимация. Москва: Мир, 1986. 318 с.

8. Голенков В. А., Морев П. Г., Радченко С. Ю. Методы математического моделирования и новые задачи ОМД. Удосконалення процесів і обладнання обробки тиском в машинобудуванні та металургї: тематич : зб. наук. пр. Краматорськ : ДДМА. 2008. С. 15-19.

9. Манилык Т., Ильин К. Практическое применение программного комплекса ABAQUS в инженерных задачах. Версия 6.5. Москва: МФТИ, ТЕСИС. 2006. 99 с.

10. Боровік П. В. Теоретичні дослідження процесів обробки металів тиском на основі методу скінчених елементів : навчальний посібник. Алчевськ: ДонДТУ. 2012. 170 с.

\section{REFERENCES}

1. Moshnin E. N. Bending and straightening on rotary machines. Moscow: Mechanical Engineering. 1967. 272 p. (in Russian).

2. Moshnin E. N. Bending and straightening machines. Moscow: MASHGIZ. 1956. 252 p. (in Russian).

3. Pasechnik N. V. Engineering encyclopedia. In 40 volumes. Vol. 4, 5 Machines and units of metallurgical production / under total. ed. V.M. Sinitskiy, N.V. Pasechnik. Moscow: Mashinostroenie. 2000. 912 p. (in Russian).

4. Guidelines for the bending of high-strength heavy plate steel on roll bending machines of metalwork factories: guide. CSRIPROJECTSTEELCONSTRUCTION. Moscow. 1978 . 61 p. (in Russian).

5. Samoilov V.A., Egorov A.B. On the relationship of force factors and curvature in the process of deformation of rolled steel by tension with bending. Izv. universities. Ferrous metallurgy. 1989. no. 7. pp. 148-149 (in Russian).

6. Satonin A.V. Numerical finite - difference mathematical modeling of the stress-strain state of the metal during the implementation of various technological schemes of pressure treatment. Improving the processes and equipment of working by pressure in mechanical engineering and metallurgy. Kramatorsk: DDMA. 2001. pp. 559-564 (in Russian).

7. Zenkevich O., Morgan K. Finite elements and approximation. Moscow: Mir. 1986. 318 p. (in Russian).

8. Golenkov V. A., Morev P. G., Radchenko S. Yu. Methods of mathematical modeling and new problems of metal forming. Improving the processes and equipment of working by pressure in mechanical engineering and metallurgy. Kramatorsk: DDMA. 2008. pp. 15-19. (in Russian).

9. Manilyk T., Ilyin K. Practical application of the ABAQUS software package in engineering problems. Version 6.5. Moscow: MIPT, TESIS. 2006. 99 p. (in Russian).

10. Borovik P. V. Theoretical advancement of metal processing processes in a vice based on the method of scraping elements: a master book. Alchevsk: DonDTU. 2012. 170 p. (in Russian).

Грибков Э. П. - д-р. техн. наук, доц. ДГМА;

E-mail: kit@dgma.donetsk.ua; https://orcid.org/0000-0002-1565-6294

Кулик А. Н. $\quad$ - канд. техн. наук, доц. ДГМА;

E-mail: amm@dgma.donetsk.ua; https://orcid.org/0000-0003-3268-6505

Гурковская С. С. - канд. техн. наук, доц. ДГМА;

E-mail: kit@dgma.donetsk.ua; http://orcid.org/0000-0001-6594-6815

Зубков В. С. - - студант ДГМА.

E-mail: amm@dgma.donetsk.ua; https://orcid.org/0000-0002-9830-6141

ДГМА - Донбасская государственная машиностроительная академия, г. Краматорск. 